\title{
Circumgalactic gas absorption in extremely metal-poor dwarf dalaxies
}

\author{
M. E. Filho ${ }^{1,2}$, J. Sánchez Almeida ${ }^{1,2}$ and C. Muñoz Tuñón ${ }^{1,2}$ \\ ${ }^{1}$ Instituto Astrofísica de Canarias, 38200 La Laguna, Tenerife, Spain \\ ${ }^{2}$ Departamento de Astrofísica, Universidad de La Laguna, 38206 La Laguna, Tenerife, Spain
}

\begin{abstract}
Accretion of metal-poor gas via cold accretion flows has been recently proposed as a means to trigger/sustain star formation in extremely metal-poor dwarf galaxies (XMPs), a scenario in agreement with theoretical predictions. We report on the tentative detection of CaII absorption used to trace the conditions of the gas clouds in the halo of the XMP UGCA 20.
\end{abstract}

Keywords. galaxies: evolution - galaxies: halos - galaxies: dwarf

The CaII doublet can be used to trace the conditions of the large-scale cold gas in the halos of nearby galaxies. We have stacked SDSS background spectra within the virial radius of each XMP (Morales-Luis et al. 2011). We report on the tentative CaII detection in the stacked spectrum of the XMP UGCA 20 (Fig. 1). The CaII lines show EWs typical of halo-type gas and a doublet ratio signaling an unsaturated system. We derive a CaII column density and mass consistent with values found for other CaII absorbers (Sardane, Turnshek \& Rao 2014). From the comparison of the various XMP components, it is plausible that the CaII originates not from star formation, but from the intergalactic medium.
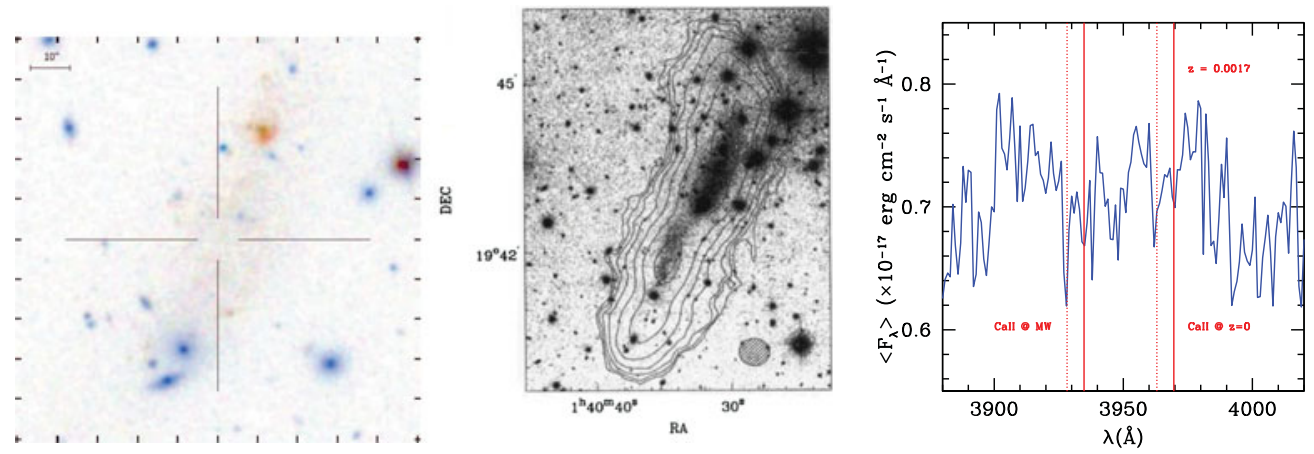

Figure 1. SDSS and HI (van Zee et al. 1996) image, and stacked spectrum of UGCA 20. Solid red lines show the CaII doublet shifted to the rest-wavelength of UGCA 20 and dotted red lines show the CaII absorption from the Milky Way.

\section{References}

Morales-Luis, A. B., Sánchez Almeida, J., Aguerri, J. A. L., \& Muñoz-Tuñón, C. 2011, ApJ, 743,77

Sardane, G. M., Turnshek, D. A., \& Rao, S. M. 2014, MNRAS, 444, 1747

van Zee, L., Haynes, M. P., Salzer, J. J., \& Broeils, A. H. 1996, AJ, 112, 129 